\title{
Preliminary analysis on linac oscillation data LI05-19 and wake field energy loss in FACET commissioning 2012
}

\author{
Yipeng Sun \\ SLAC National Accelerator Laboratory, Menlo Park, California 94025, USA
}

July 23, 2012

\begin{abstract}
In this note, preliminary analysis on linac oscillation data in FACET linac LI05-09 plus LI11-19 is presented. Several quadrupoles are identified to possibly have different strength, compared with their designed strength in the MAD optics model. The beam energy loss due to longitudinal wake fields in the S-band linac is also analytically calculated, also by LITRACK numerical simulations.
\end{abstract}

\section{Preliminary analysis on oscillation data}

To benchmark the FACET linac machine with its design MAD [1] optics, linac betatron oscillation data is measured and analyzed. This technique is routinely used to improve the performance of SLC linac [2]. The FACET linac LI05-19 is divided into 5 sub sections. In each section, two sets of betatron oscillation data are taken in horizontal and vertical plane respectively, which is done by changing two steering dipole magnets XCOR202 and YCOR203 (near the starting point of each section) in a range of -0.01 to $0.01 \mathrm{kG}$.m with 11 steps. The BPM readings are then recorded in each section for each step of steering dipole magnets setting.

The TWISS parameters at all quadrupoles, BPMs and certain dipole steering magnets are generated in MAD [1]. The measurement data is taken in SLAC MCC using the online control program SCP. Several scripts are prepared and saved in SCP (and its default directory), such as the button 'yipeng_s7_9', 'yipeng_s11_13', 'yipeng_s14_16' and 'yipeng_s17_19'. The data postprocessing (AWK scripts), the calculation of oscillation data from the MAD model and other parts of algorithms are all coded in MATLAB.

The difference of any two sets of BPM readings denotes a response of the linac optics to the initial beam angle (excited by the steering dipole magnets mentioned above), which already subtracts the contribution of the downstream steering dipole magnets. The contribution on the BPM readings from the downstream steering dipole magnets are the same between different sets of data, as the strength of the downstream steering dipole magnets do not change.

The agreement between the FACET linac machine setting and its MAD optics model is good in LI05-06 and LI14-16, as shown in Figure 1 and Figure 7 below. 
There are certain differences in the betatron phase advance between the FACET linac machine setting and its MAD optics model in LI07-09, LI11-13 and LI17-19, as shown in Figure 2, Figure 4 and Figure 8 below. By tuning some quadrupole strength in the MAD optics model, the model could fit to the measurement data, as shown in Figure 3, Figure 6 and Figure 9 below.

As shown in Figure 5, in MATLAB script the two steering dipole magnets Li11XCOR202 and Li11YCOR203 are scanned in a series of strength, $\theta=15 \mu \mathrm{rad}, \theta=20 \mu \mathrm{rad}, \theta=25 \mu \mathrm{rad}, \theta=30 \mu \mathrm{rad}$, $\theta=40 \mu \mathrm{rad}, \theta=50 \mu \mathrm{rad}$, and $\theta=60 \mu \mathrm{rad}$. It is observed that the launching steering dipole magnet's strength only affects the oscillation data amplitude, and it does not change the betatron phase advance of the oscillation. The difference in betatron phase advance should be from the quad strength error, and other effects such as transverse wake fields.

In Table 1 the suspicious quadrupoles are listed with the possible strength error from measurement data fit.

Table 1: Quad error from measurement data fit.

\begin{tabular}{c|c|c}
\hline & MAD strength $\mathrm{K} 1\left[\mathrm{~m}^{-1}\right]$ & Fit strength $\mathrm{K} 1\left[\mathrm{~m}^{-1}\right]$ \\
\hline Q070401T & 1.44 & 1.24 \\
Q110301T & -1.16 & -0.80 \\
Q110501T & -1.41 & -1.00 \\
Q110601T & 1.10 & 1.00 \\
Q110801T & 1.15 & 0.95 \\
Q180501T & -0.49 & -0.99 \\
\hline
\end{tabular}

Possible error sources are:

- quadrupole strength error (systematic and random error)

- dipole corrector strength scaling error

- BPM random error (resolution, roughly $50 \mu \mathrm{m}$ for SLAC linac)

- BPM reading scaling error (systematic error)

- Effects not included in the algorithm, wake fields, RF kicks etc.

- Beam jitter (unstable initial angle)

\section{$2 \quad$ Wake field induced energy loss}

The electron bunch loses energy due to longitudinal wake fields when it is accelerated in the long linac accelerator. Using short-range approximation of longitudinal wake field, the acceleration structure parameters and the electron beam parameters, the energy loss factor per unit length and unit charge can be calculated and denoted as [3]

$$
k\left(\sigma_{z}\right) \approx \frac{Z_{0} c}{2 \pi a^{2}} e^{-0.88 \sqrt{\sigma_{z} / s_{0}}}
$$


Considering both RF acceleration and wake fields induced energy loss, the net energy gain of an electron bunch is [3]

$$
\Delta E=E_{a c c} \cos \phi e^{-k_{r f}^{2} \sigma_{z}^{2} / 2}-e N L k\left(\sigma_{z}\right)
$$

The final beam energy at linac end is calculated numerically with LITRACK and shown in Figure 10 (left), with and without longitudinal wake fields. A comparison between LITRACK calculated energy loss and analytically estimated energy loss from formula 2 is shown in Figure 10 (right), where good agreement is achieved.

LiTrack 1D simulation conditions: $2 \times 10^{10}$ electrons in uniform distribution, accelerated from 9 $\mathrm{GeV}$ to $20 \mathrm{GeV}$ in $550 \mathrm{~m}$ SLAC S-band RF (on crest 0 degree), intrinsic energy spread $5 \times 10^{-4}$.

The energy loss can be further expressed as a function of average RF phase in LI04-09, as shown in Figure 11. The bunch length is analytically calculated up to third order as shown in formula 3 [4]. Keep sector 10 chicane energy at $9 \mathrm{GeV}$, with a linear momentum compaction of R56 $=-76 \mathrm{~mm}$. The result shows rough estimate with $450 \mathrm{~m}$ S-band RF from $1.2 \mathrm{GeV}$ to $9 \mathrm{GeV}$, with an initial bunch length of $1.2 \mathrm{~mm}$. A part in bunch head and tail is cut to minimize the difference between Gaussian-fit and RMS bunch length, which is due to second order and higher order effects.

$$
\sigma_{z}^{2}=a^{2} R_{56}^{2} \sigma_{\delta i}^{2}+\left(1+h_{1} R_{56}\right)^{2} \cdot \sigma_{z, i}^{2}+3 \cdot\left(h_{1}^{2} \cdot T_{566}+h_{2} \cdot R_{56}\right)^{2} \cdot \sigma_{z, i}{ }^{4}
$$

where $\sigma_{\delta i}$ denotes the initial un-correlated RMS energy spread, $\sigma_{z, i}$ initial bunch length.

\section{Acknowledgement}

The author would like to thank C. Adolphsen, F.-J. Decker, A. Latina, N. Lipkowitz, Y. Nosochkov, U. Wienands, M. Woodley, G. Yocky for helpful discussions. Special thanks go to C. Adolphsen for his guidance and support.

This work was supported by the DOE under Contract DE-AC02-76SF00515.

\section{References}

[1] H. Grote, F.C. Iselin, "The MAD Program (Methodical Accelerator Design) Version 8.15," CERN/SL/90-13 (AP) (1990).

[2] Chris Adolphsen et al., SLAC-PUB-5581 (1991).

[3] Karl Bane et al., in Proceedings of the 2003 Particle Accelerator Conference, page 3126-3128, http://accelconf.web.cern.ch/AccelConf/p03/PAPERS/RPPB054.pdf (2003).

[4] Yipeng Sun et al., SLAC-PUB-14511 (2012). 

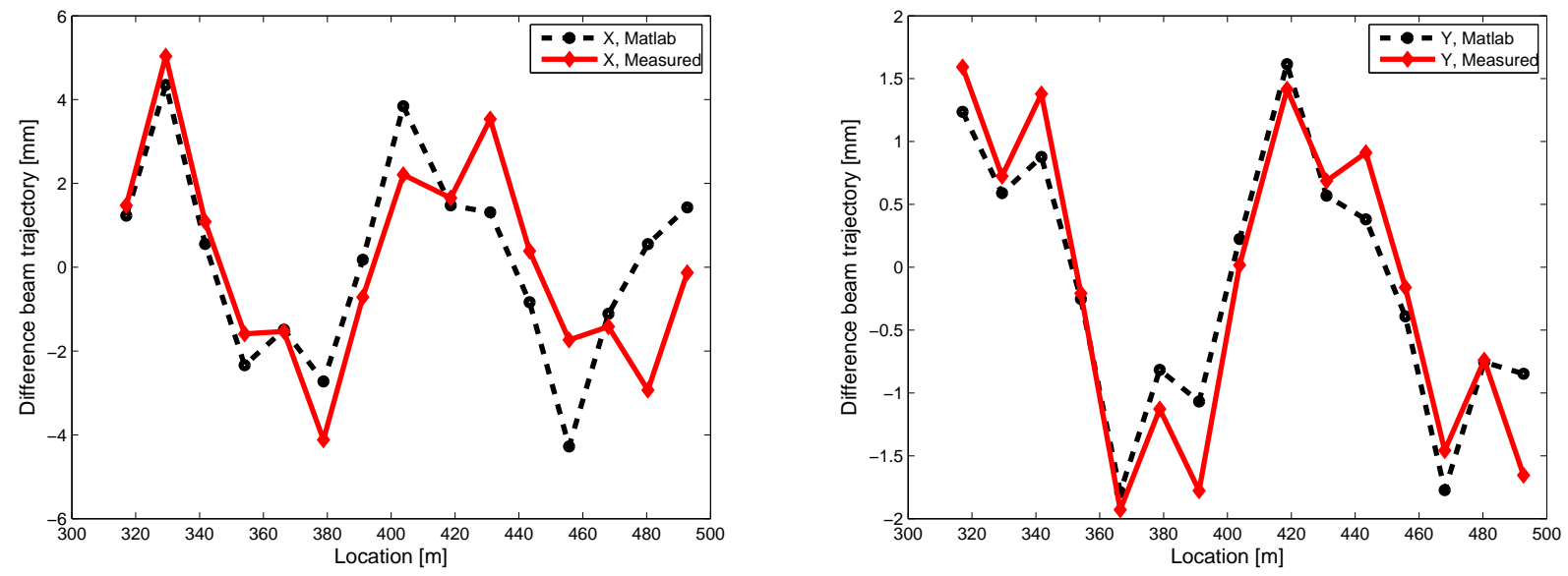

Figure 1: Benchmark of FACET linac machine LI05-06 with its MAD optics model, using linac oscillation data measured by changing two steering dipole magnets Li05XCOR202 and Li05YCOR203 (range -0.01 to 0.01 kG.m, in 11 steps). Left: horizontal plane; Right: vertical plane.
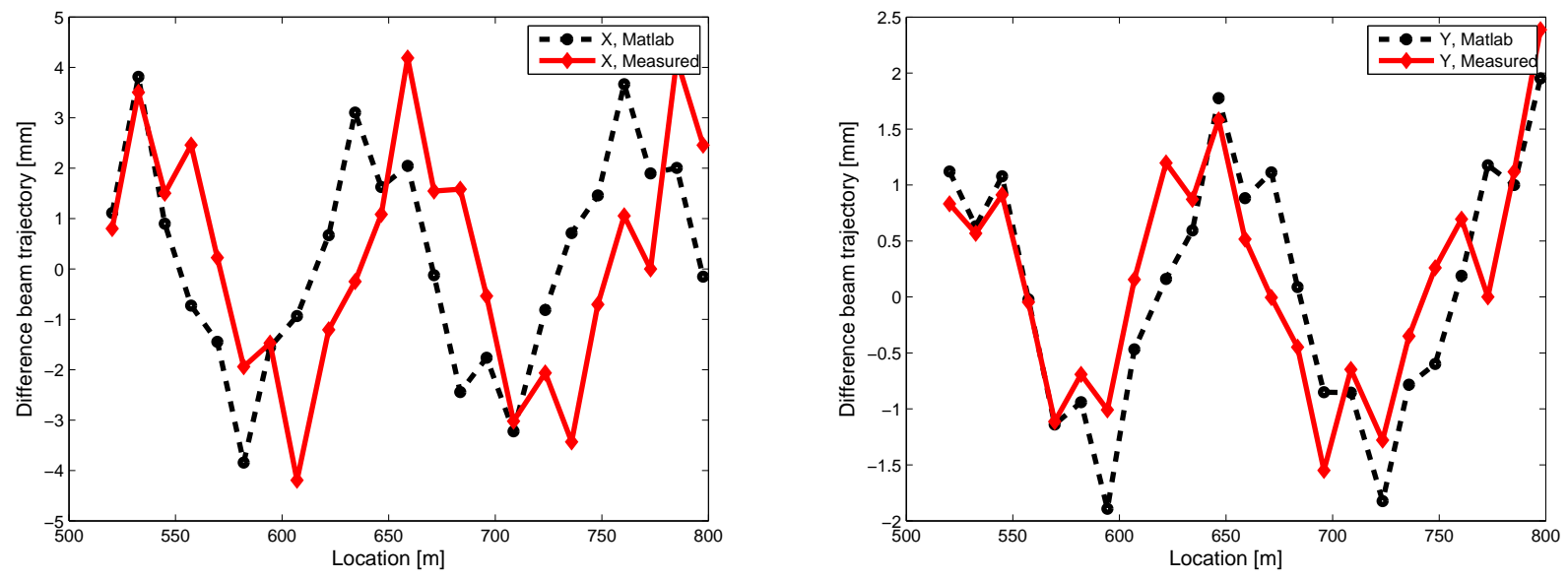

Figure 2: Benchmark of FACET linac machine LI07-09 with its MAD optics model, using linac oscillation data measured by changing two steering dipole magnets Li07XCOR202 and Li07YCOR203 (range -0.01 to $0.01 \mathrm{kG} . \mathrm{m}$, in 11 steps). Left: horizontal plane; Right: vertical plane. 

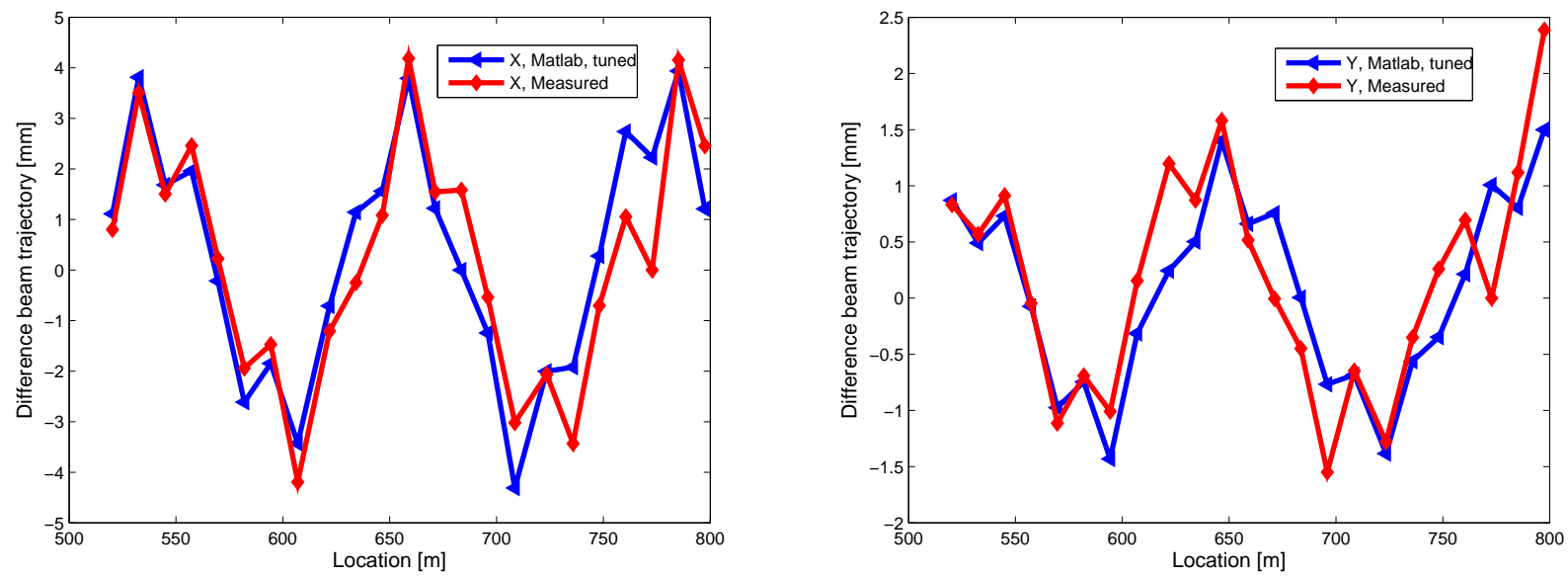

Figure 3: Benchmark of FACET linac machine LI07-09 with its tuned MAD optics model, using linac oscillation data measured by changing two steering dipole magnets Li07XCOR202 and Li07YCOR203 (range -0.01 to $0.01 \mathrm{kG} . \mathrm{m}$, in 11 steps). The quadrupole Q070401T is changed from $K 1=1.44$ to $K 1=1.24$ in MAD optics model. The steering dipole magnets LiOrYCOR203 is changed from $\theta=45 \mu \mathrm{rad}$ to $\theta=35 \mu \mathrm{rad}$ in $M A D$ optics model. Left: horizontal plane; Right: vertical plane.
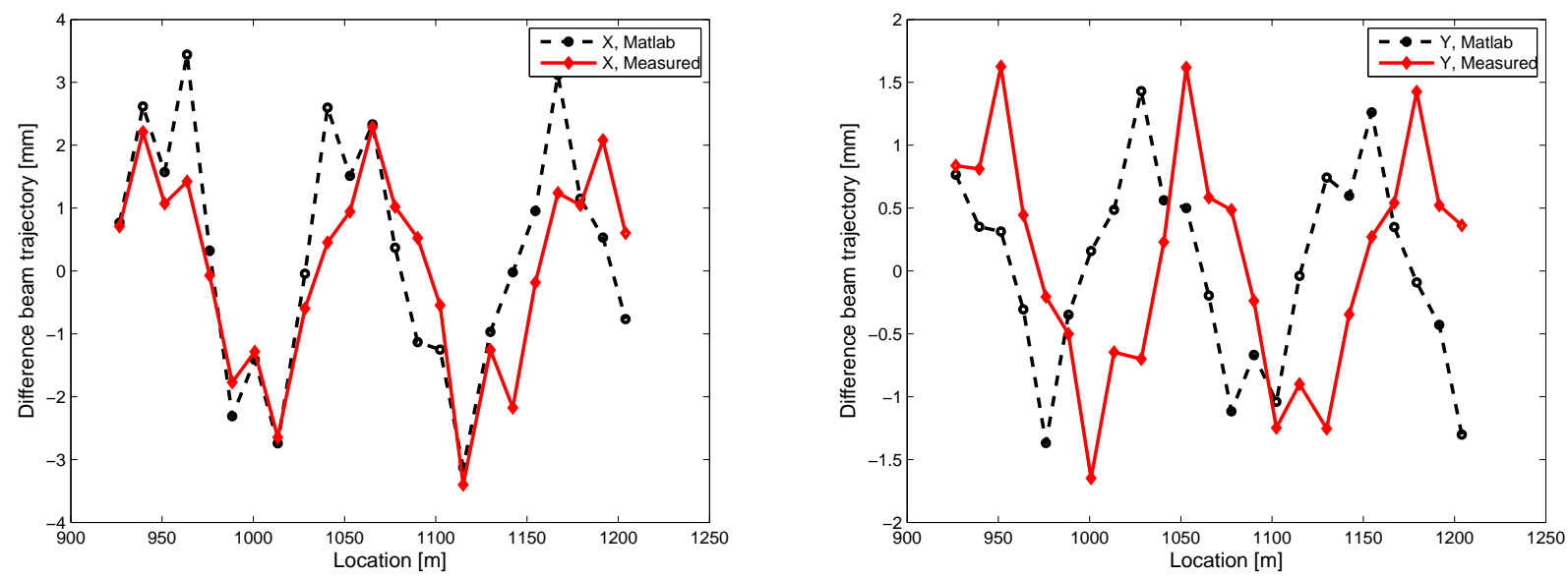

Figure 4: Benchmark of FACET linac machine LI11-13 with its MAD optics model, using linac oscillation data measured by changing two steering dipole magnets Li11XCOR202 and Li11YCOR203 (range -0.01 to $0.01 \mathrm{kG} . \mathrm{m}$, in 11 steps). Left: horizontal plane; Right: vertical plane. 

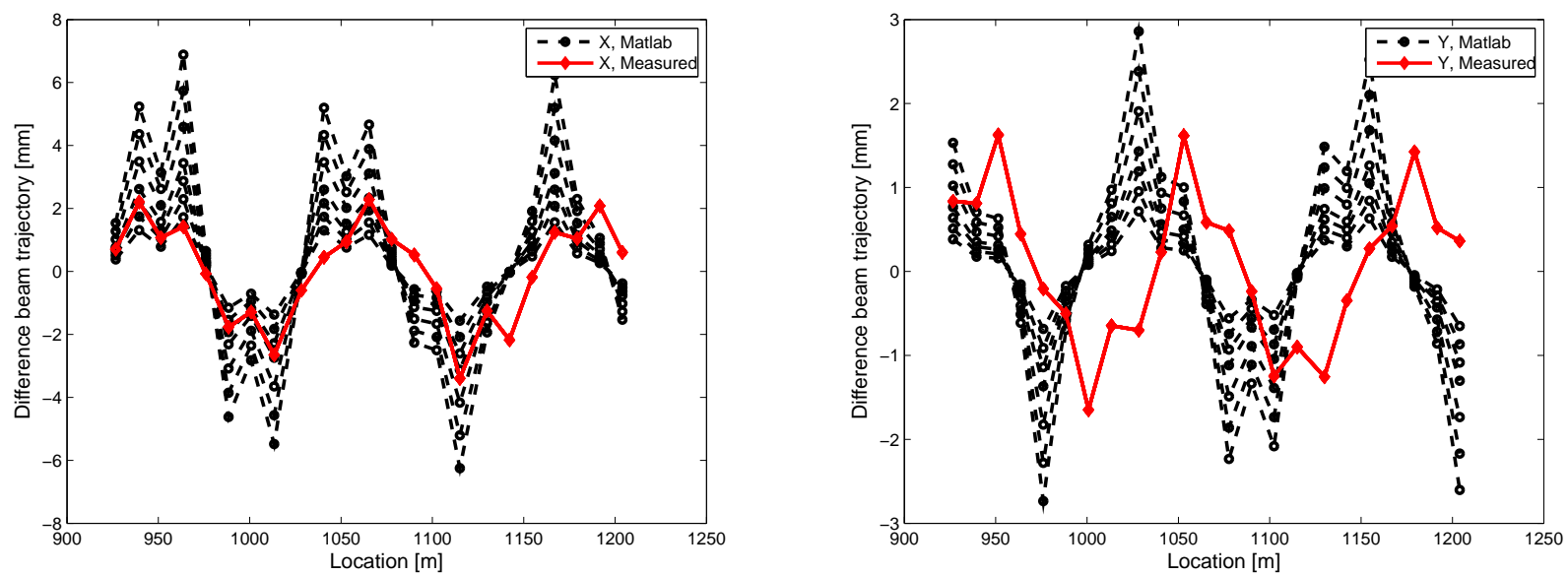

Figure 5: Benchmark of FACET linac machine LI11-13 with its MAD optics model, using linac oscillation data measured by changing two steering dipole magnets Li11XCOR202 and Li11YCOR203 (range -0.01 to $0.01 \mathrm{kG} . \mathrm{m}$, in 11 steps). In MATLAB script the two steering dipole magnets Li11XCOR202 and Li11YCOR203 are scanned in a series of strength, $\theta=15 \mu \mathrm{rad}, \theta=20 \mu \mathrm{rad}$, $\theta=25 \mu \mathrm{rad}, \theta=30 \mu \mathrm{rad}, \theta=40 \mu \mathrm{rad}, \theta=50 \mu \mathrm{rad}$, and $\theta=60 \mu \mathrm{rad}$. It is observed that the launching steering dipole magnet's strength only affects the oscillation data amplitude, and it does not change the betatron phase advance of the oscillation. Left: horizontal plane; Right: vertical plane.
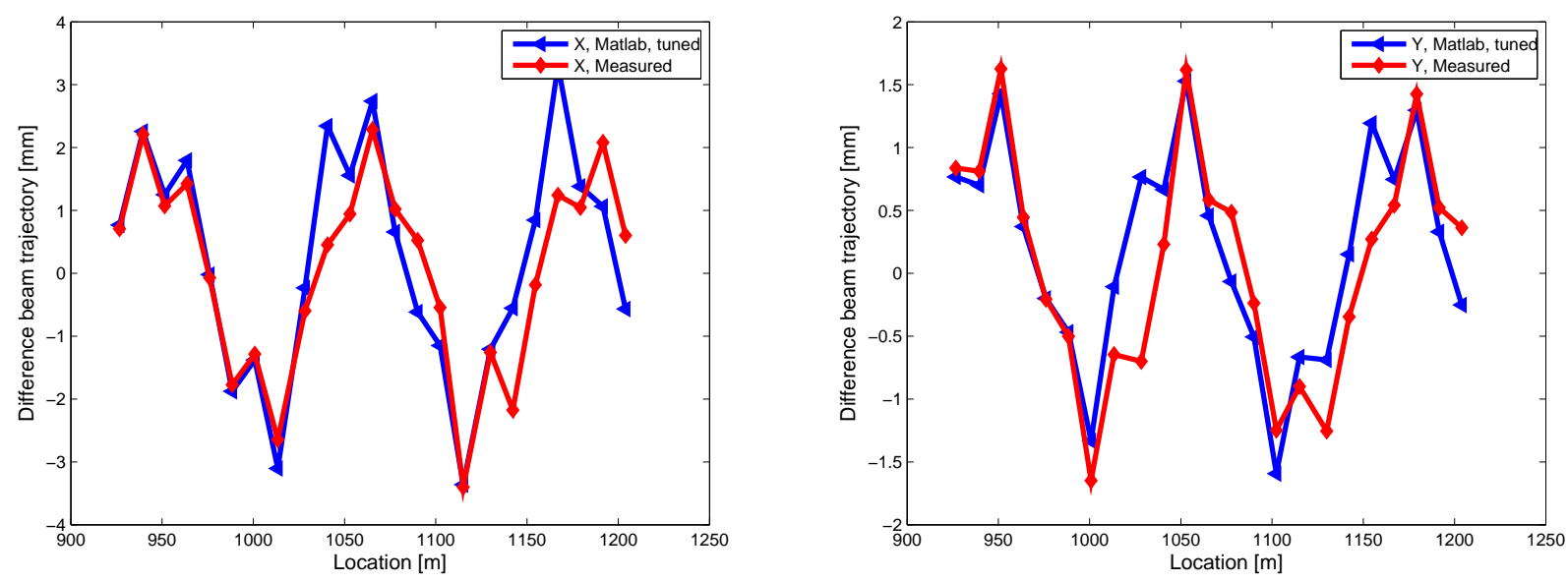

Figure 6: Benchmark of FACET linac machine LI11-13 with its tuned MAD optics model, using linac oscillation data measured by changing two steering dipole magnets Li11XCOR202 and Li11YCOR203 (range -0.01 to $0.01 \mathrm{kG} . \mathrm{m}$, in 11 steps). The quadrupole Q110301T is changed from $K 1=-1.16$ to $K 1=-0.80$ in MAD optics model. The quadrupole Q110501T is changed from K1=1.41 to $K 1=-0.95$ in MAD optics model. The quadrupole Q110601T is changed from K1=1.10 to $K 1=1.00$ in MAD optics model. The quadrupole Q110801T is changed from K1=1.15 to K1=0.95 in MAD optics model. Left: horizontal plane; Right: vertical plane. 



Figure 7: Benchmark of FACET linac machine LI14-16 with its MAD optics model, using linac oscillation data measured by changing two steering dipole magnets Li14XCOR202 and Li14YCOR203 (range -0.01 to $0.01 \mathrm{kG} . \mathrm{m}$, in 11 steps). Left: horizontal plane; Right: vertical plane.


Figure 8: Benchmark of FACET linac machine LI17-19 with its MAD optics model, using linac oscillation data measured by changing two steering dipole magnets Li17XCOR202 and Li17YCOR203 (range -0.01 to $0.01 \mathrm{kG} . \mathrm{m}$, in 11 steps). Left: horizontal plane; Right: vertical plane. 

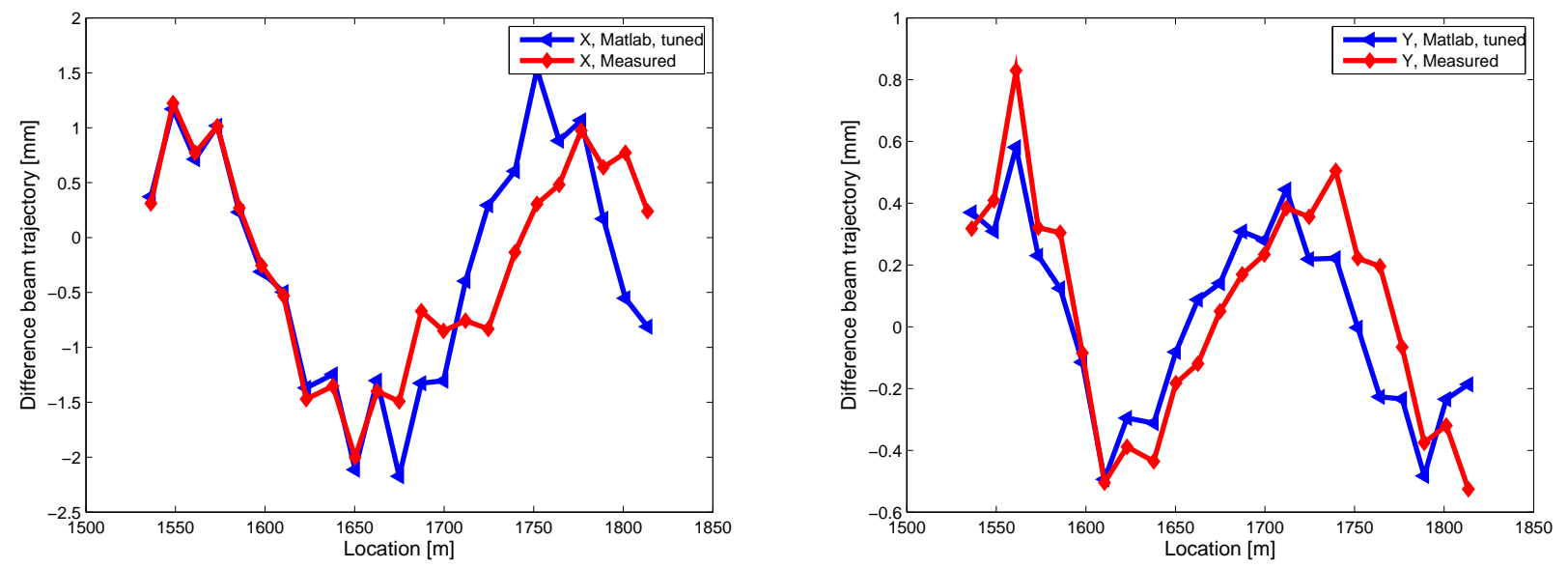

Figure 9: Benchmark of FACET linac machine LI17-19 with its tuned MAD optics model, using linac oscillation data measured by changing two steering dipole magnets Li17XCOR202 and Li17YCOR203 (range -0.01 to $0.01 \mathrm{kG} . \mathrm{m}$, in 11 steps). The quadrupole Q180501T is changed from $K 1=-0.49$ to $K 1=-0.99$ in MAD optics model. Left: horizontal plane; Right: vertical plane.


Figure 10: Left: final beam energy at FACET linac end as a function of bunch length in linac LI1119; Right: wake field induced energy loss as a function of bunch length. LiTrack 1D simulation conditions: $2 \times 10^{10}$ electrons in uniform distribution, accelerated from $9 \mathrm{GeV}$ to $20 \mathrm{GeV}$ in $550 \mathrm{~m}$ SLAC S-band RF (on crest 0 degree), intrinsic energy spread $5 \times 10^{-4}$. 


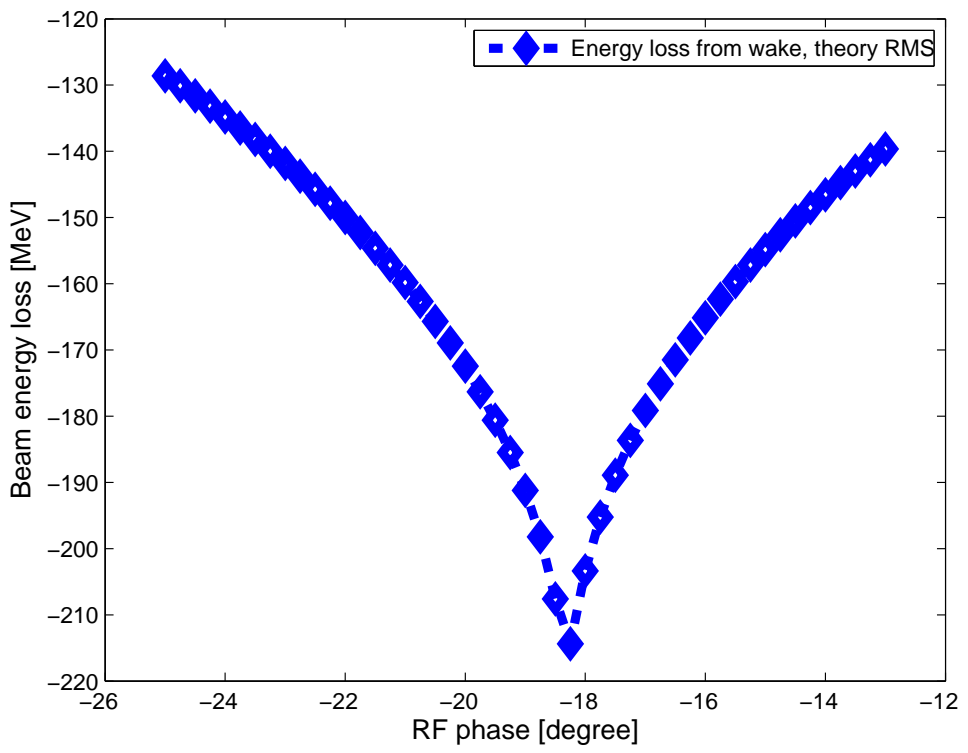

Figure 11: Analytically estimated wake field induced energy loss as a function of average RF phase in LI04-09. LiTrack 1D simulation conditions: $2 \times 10^{10}$ electrons in uniform distribution, accelerated from $9 \mathrm{GeV}$ to $20 \mathrm{GeV}$ in $550 \mathrm{~m}$ SLAC S-band RF (on crest 0 degree), intrinsic energy spread $5 \times 10^{-4}$. Keep sector 10 chicane energy at $9 \mathrm{GeV}$, with a linear momentum compaction of R56= $-76 \mathrm{~mm}$. The result shows rough estimate with $450 \mathrm{~m}$ S-band RF from $1.2 \mathrm{GeV}$ to $9 \mathrm{GeV}$, with an initial bunch length of $1.2 \mathrm{~mm}$. A part in bunch head and tail is cut to minimize the difference between Gaussian-fit and RMS bunch length, which is due to second order and higher order effects. 